DOI 10.37882/2223-2974.2021.02.09

\title{
ПЕРСОНАЛИЗАЦИЯ КАК ВЕРОЯТНОЕ БУДУЩЕЕ КОРПОРАТИВНОЙ ОТЧЕТНОСТИ
}

\section{PERSONALIZATION AS A PROBABLE FUTURE CORPORATE REPORTING}

\section{Zh. Kashchina}

Summary: The article discusses the general problems of corporate reporting methodology and forecasting methods based on credentials. Special attention is paid to the problem of the contradiction between the interests of issuers and the existing information requests of users of public reporting. Ways of resolving these contradictions are proposed. In particular, the article discusses alternative methods of corporate reporting, on the basis of which it would be possible to build more accurate forecasts about the future performance and financial position of organizations. The relevance of the proposed methods is confirmed by the results of the research. The theoretical basis for the study was the work devoted to such scientific areas as isomorphism, neoinstitutionalism, as well as the theory of signals.

We can observe the convergence of methods and technologies that were once isolated from each other. In addition, advances in many areas of modern science are associated, among other things, with increased computing capabilities. This also applies to the processing of data used to make economic decisions by business, the basis of which is largely accounting. One of the possible results of these trends is the replacement of static financial statements with the original (primary) data that can be obtained and analyzed by the end user.

Keywords: accounting, corporate reporting, personalized reporting, isomorphism, signal theory, neoinstitutionalism, theory of fields.

\section{НеАостатки трациционных форм корпоративной отчетности}

2 вучащие сегодня мнения относительно возможного влияния технологического развития на судьбу бухгалтерского учета весьма неоднозначны. Кто-то воспринимает новые технологии как новые возможности совершенствования процесса коммуникации компаний и их стейкхолдеров, кто-то, наоборот, видит в цифровизации учета прямую угрозу бухгалтерской профессии. С одной стороны, нельзя не согласиться с замечанием о том, что сегодняшняя цифровизация учета - это всего лишь избавление бухгалтера от рутинной работы. С другой стороны, технические новации снимают вопрос ретроспективности информации, предоставляемой заинтересованным лицам, принимающим инвестиционные и управленческие решения, а также значимо усиливают аналитические функции учета, перенося внимание бухгалтера в область оценки, формируемых програм-
Кащина Жанна Евгеньевна

Аспирант, Санкт-Петербургский государственный университет z.kaschina@lsg.spb.ru

Аннотация: В статье рассматриваются общие проблемы методологии корпоративной отчетности и методов прогнозирования на основе учетных данных. Особое внимание уделено проблеме противоречия между интересами эмитентов и существующими информационными запросами пользователей публичной отчетности. Предложены пути разрешения этих противоречий. В частности, в статье рассматриваются альтернативные методы представления корпоративной отчетности, на основе которых можно было бы строить более точные прогнозы о будущих результатах деятельности и финансовом положении организаций. Актуальность предложенных методов подтверждена результатами проведенного исследования. Теоретической основой для исследования послужили работы, посвященные таким научным направлениям как изоморфизм, неоинституционализм, а также теории сигналов.

Ключевые слова: учет, корпоративная отчетность, персонализация, изоморфизм, теория сигналов, неоинституционализм, теория полей.

мой данных и их анализа. Необходимо отметить, что потребность в информационных продуктах современных интернет-технологий, в частности, в таких как персонализированная отчетность, предоставляемая в реальном времени, не нова. К устранению таких недостатков корпоративной отчетности как ретроспективность, неинформативность, агрегированность и т. п. стремились пользователи отчетности еще до появления интернета.

Тем не менее, по-прежнему одним из основных вопросов, обсуждаемых в последние годы в литературе в области бухгалтерского учета, является использование учетных данных при принятии инвестиционных и управленческих решений (Дамодаран, Джонсон, Маттесич, Сортер, Стаубус, Флегм, Хендриксен, Ван Бреда, Чамберс, Ковалев В.В., Пятов М.Л., Соколов Я.В. и другие).

Несмотря на критику содержащихся в публичной отчетности сведений, она остается важным официальным 
источником информации о положении дел компании. Говоря о роли учетных данных в экономике, можно привести слова Ковалева В.В.и Ковалева Вит.В., которые писали, что информация, генерируемая в системе бухгалтерского учета занимает существенное место в информационном обеспечении экономик и является своеобразным средством коммуникации. Отсюда возникает проблема адекватности алгоритмов производства и способов интерпретации и восприятия отчетных данных $[8$, с 3].

Многие авторы (Стемп, Мамфорд, Писнел, Маттесич ) [38, с. 158] всегда сходились во мнении, относительно основной цели корпоративной отчетности, заключающейся в предоставлении актуальной и достоверной информации о реальных показателях деятельности предприятия всем заинтересованным пользователям, нуждающимся в такой информации для принятия инвестиционных и управленческих решений.

В литературе, посвященной учету, часто можно встретить критику существующих форм корпоративной отчетности. При этом, совершенно определенно можно сказать, что с учетом уровня развития современных технологий, такие недостатки как ретроспективность, унификация, неинформативность, содержащихся в отчетах данных, носят чисто технический характер.

Так можно сделать предположение о том, что такой вид информации как ретроспективные данные традиционной публичной отчетности не могут являться определяющим фактором, способным влиять на ожидания участников рынка о состоянии экономики, финансовом положении компаний, будущих доходах и рисках, что противоречит основной цели корпоративной отчетности. Кроме того, не смотря на многочисленные исследования, по-прежнему нет полной ясности в вопросе о взаимосвязи между традиционной финансовой отчетностью компании и решениями, принимаемыми на рынке капитала, что вообще ставит под сомнение востребованность некоторых данных, содержащихся в корпоративной отчетности.

Другим существенным недостатком современной корпоративной отчетности, по мнению некоторых авторов, является существующие методы расчета бухгалтерской прибыли. Основанием для данного утверждения является, в частности, тот факт, что бухгалтерская прибыль, которая представлена в современной финансовой отчетности, в экономическом смысле некорректна, так как в ней смешаны логические принципы двух антагонистических балансовых концепций: динамического и статического балансов [6, с.194].

Кроме того, по мнению Пятова М.Л. и Соколовой Н.А., конфликт интересов пользователей бухгалтерской информации заключается в противоречии задач представ- ления информации о платежеспособности и рентабельности компаний. Реализация этих двух противоречащих друг другу целей предполагает совершенно разные подходы к оценке деятельности компаний [16,с. 352] .

В настоящее время агрегирование и усреднение учетных данных - это артефакты ушедшей эпохи медленного сбора и высоких затрат на передачу данных. Современные учетные системы позволяют обрабатывать и хранить значительный объем первичных данных, которые могли бы быть полезны и доступны внешним пользователям. Та информация, которая содержится в существующей публичной отчетности, состоит из одного статического набора данных, при этом предназначена удовлетворить запросы различных групп пользователей. Это приводит к асимметрии информации, и тому, что опускаются многие виды данных, влияющие на оценку и управленческие решения.

Однако, безусловная однозначность в формировании картины имущественного и финансового положения фирмы возможна лишь в том случае, если будут точно регламентированы все возможные операции, а также задействованные в них оценки и методы отражения этих операций в учете. Мэтьюс М. и Перера М. считали, что подобная ситуация вряд ли возможна на практике $и$ кроме того, строгое регулирование учета привело бы к появлению нескончаемого ряда инструкций для каждой конкретной ситуации. Это свело бы функции бухгалтера к слепому следованию правилам и исключило бы аспект профессионального суждения, столь важного для nрофеeccuu [11, с. 469].

Очевидно, что проблема агрегированности связана прежде всего с тем, что информация о фактах хозяйственной жизни на пути от первичных документов до финансовой отчетности претерпевает ряд преломлений [2, с. 175]. В результате чего пользователь получает лишь отдаленное представление о реальном состоянии дел компании.

В этой связи, можно предположить, что «модель текущей финансовой отчетности уже не соответствует ее главной цели: она не позволяет оценить качество и устойчивость финансового положения - отсутствует информация предвидения (прогнозирования)». [13, с. 10]

\section{Путь решения - персонализашия}

В современном мире информация все чаще становится предметом экономической деятельности, целью которой является воздействие на потребителя. Речь, в частности, идет о создании технологий обработки «больших данных». В этих условиях отчетность, ориентированная на конкретного пользователя (персонализированная), может стать важной частью конкурентного преиму- 
щества любой компании. Так, по мнению М.Л. Пятова и В.В. Глинского «Персонифицированность отчетных данных, заключающаяся в их настройке под запросы конкретного пользователя, - это также воплощающаяся реальность текущего момента времени.» $[15$, с. 8]

Под персонализированной отчетностью, в контексте данного исследования, мы будем понимать, как финансовые, так и не финансовые (агрегированные или первичные) данные, объединенные в виде формализованного отчета, содержание которого определяется соглашением между пользователем такой отчетности и предоставляющей ее стороной.

Безусловно, идея детализации определенной части данных отчетности в интересах тех или иных групп пользователей не нова. В настоящее время она реализуется с одной стороны в пояснениях к балансу, с другой - в раскрытии специфики методологии в пояснительной записке. Кроме того, все развитие публичной отчетности характеризуется с одной стороны выбором и утверждением детализации ее статей и в то же время их унификацией, с другой стороны. Развитие технологий и динамики информационных запросов способствует персонализации или индивидуальной детализации данных отчетности.

Основными принципами «бумажной парадигмы» корпоративной отчетности являются публичность и стандартизация. Однако, современные интернет-технологии создают условия для полной индивидуализации (персонализации) корпоративной отчетности, приспособления ее к персональным нуждам конкретного потребителя. Структурированная информация в сочетании со способностью аналитических и поисковых систем находить и интерпретировать нужные данные позволяют любому пользователю составить собственный «отчет» о результатах деятельности компании на любую отчетную дату. Таким образом, пользователи могут строить собственные прогнозы исходя из различных сценариев и предпосылок относительно темпов роста дохода, себестоимости продукции компании и множества других меняющихся показателей ее деятельности.

В этой связи будет уместным привести слова Хендриксена и Ван Бреды, которые еще 1992 году писали: «На смену бухгалтерским регистрам придут базы данных, частью которых станет финансовая информация. Использование структурных гипертекстов позволит пользователю добраться до информачии любого уровня детализации, которая может ему понадобиться для анализа. Со всей той информацией «на кончиках пальцев» инвесторов и совершится реальная революция в бухгалтерском учете. Все что для этого необходимо, -принять новую технологию». [21, с.37]

Сложно спорить с утверждением, что бухгалтерская информация, такая, как, например, данные баланса и отчеты о прибылях и убытках, является несовершенной. Как уже упоминалось, содержание финансовой отчетности страдает от таких проблем, как неоднозначная и неоднородная методология, ретроспективность. Однако, если бы фирмы готовы были полностью или частично открыть доступ к своей внутренней учетной информации, это бы значительно повысило качество анализа, проводимого пользователями, повысило доверие к содержанию отчетности, а также упростило процесс верификации транзакций [26, с. 12].

В настоящее время согласно п. 4 Приказа Минфина России от 02.07.2010 N 66н «О формах бухгалтерской отчетности» нормативным регулированием бухгалтерского учета не предусмотрена четкая структуризация информации, формируемой в пояснениях к бухгалтерскому балансу и отчету о прибылях и убытках. Принято считать, что содержание пояснений определяется организациями самостоятельно в произвольной форме. Действующие нормативные акты содержат лишь общие требования к ее содержанию. Однако они неоднородны и если какое-либо требование отдельного ПБУ не реализовано в таблицах - пояснениях, рекомендованных Минфином России, то, следовательно, данное раскрытие организация должна дать самостоятельно, для чего разработать таблицу - пояснение или раскрыть требуемую информацию текстом. При этом, также подразумевается, что раскрываемая в пояснениях информация должна полностью удовлетворять требованиям и запросам всех групп пользователей отчетности, что на практике реализовать практически невозможно.

В начале исследования автором были поставлены следующие вопросы:

- Существует ли на практике потребность в персонализированной корпоративной отчетности?

- Каково содержание действительных информационных запросов конкретных групп пользователей отчетности, не удовлетворяемых ее существующим (общим) форматом?

- На сколько развитие практики персонализированной отчетности обеспечивается современным уровнем реализуемых в экономической деятельности технологий?

В целях определения содержания действительных информационных запросов конкретных групп пользователей отчетности, не удовлетворяемых ее существующим (общим) форматом было проведено исследование, материалом для которого послужило более 100 различных формализованных запросов пользователей отчетности: тендерные таблицы; требования и письма контролирующих органов; внутренние положения и документы, касающиеся «скоринга» заемщиков кредитных организаций; запросы различных кредиторов, направ- 
ляемые в рамках соблюдения должной осмотрительности. Репрезентативность данных подтверждается тем, что в ходе исследования в равной степени были проанализированы запросы всех основных групп пользователей корпоративной отчетности.

В ходе исследования были проанализированы действительные формализованные запросы пользователей отчетности различных компании с целью изучения информационных требований, предъявляемых заинтересованными сторонами.

Таким образом, результаты исследования подтверждают, гипотезу о том, что сегодня со стороны пользователей учетной и отчетной информации существует высокая заинтересованность в доступе к первичной информации о фактах хозяйственной жизни компании в режиме реального времени, при этом интегрированные интернет-технологии могут способствовать значительному повышению доверия к бизнесу и закреплению, таким образом, за персонализированной отчетностью статуса конкурентного преимущества.

Следует также отметить, что проведенное исследование, прежде всего, позволило подтвердить предположение о том, что формализация форм отчетности в условиях растущей автоматизации теряет свою значимость, а также констатировать будущую неотвратимость перехода от анализа выборки к анализу большего объема данных.

В результате проведенного исследования было обосновано предположение о том, что подавляющее большинство пользователей отчетности (кредиторы, контрагенты, контролирующие органы и т.д.) заинтересованы в получении первичной информации о фактах хозяй- ственной жизни компании. Из чего, в частности, можно сделать вывод, что в настоящее время самостоятельно проводить анализ на основе первичной информации предпочитают большинство пользователей финансовой отчетности.

Исследование показало, что в доступе к нефинансовой информации и данным управленческого учета при проверке контрагентов нуждается практически 100\% заказчиков и покупателей товаров и услуг. Кроме того, при оценке заемщика банки регулярно предъявляют требование о раскрытии нефинансовой информации о текущей деятельности предприятия, а также о предоставлении первичной информации. Следовательно, не смотря на тот факт, что многими компаниями составляется интегрированная отчетность, пользователям интересны, именно, актуальные первичные данные на основе которых она формируется.

При этом, данные исследования так же показали, что не смотря на то, что большинство пользователей отчетности признают, что повышение информационной прозрачности (предоставление рынку большего количества интересующей его информации) влечет за собой совершенно очевидные выгоды для компании, в настоящее время абсолютное меньшинство эмитентов отчетности готовы открыть пользователям доступ к первичным учетным данным компании. Главным образом это связано с отсутствием соответствующего регулирующего, подобный информационный обмен, законодательства, а также с расхождением в восприятии информационной прозрачности между пользователями и эмитентами. Так, в ходе исследования выяснилось, что большинство компаний склонны оценивать свои усилия по удовлетворению информационных потребностей пользователей гораздо выше, чем их оценивает рынок.

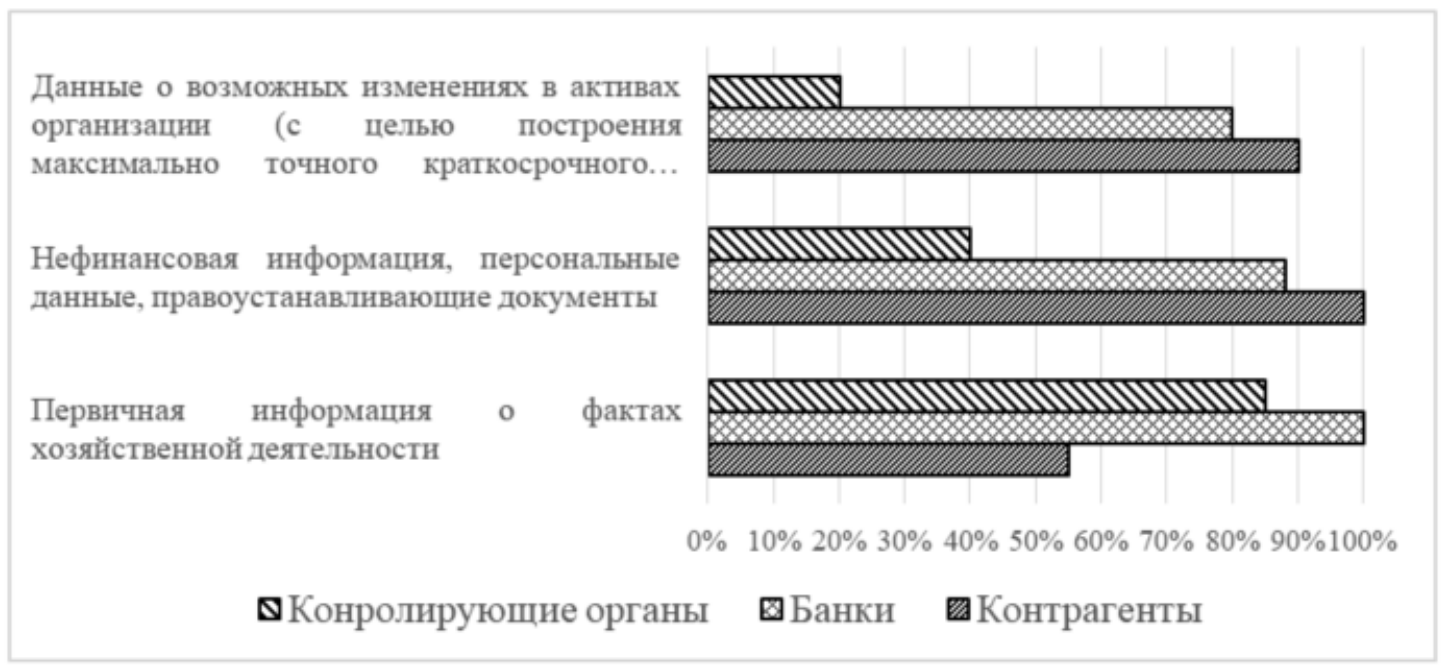

Рис. 1. Основные группы дополнительных формализованных запросов пользователей корпоративной отчетности Источник: авторская разработка (Персонализированная корпоративная отчетность: принципы и перспективы развития. Международный бухгалтерский учет. - 2019. - Т. 22. № 2 (452). - С. 147-157.) 


\section{Теория сигналов и теория изоморфизма в контексте прочесса коммуникашии эмитентов и пользователей отчетности}

Действительно, современные интернет-технологии открывают компаниям безграничные возможности совершенствования своей отчетной практики. В этой связи, можно предположить, что стандарты финансовой отчетности, которые регулируют представление и агрегирование данных должны измениться, а существующие возможности анализа «больших данных» требуют новой парадигмы корпоративной отчетности, ориентированной на удовлетворение индивидуальных запросов пользователей в режиме реального времени.

Оценить вероятность такой практики, по нашему мнению, позволяют, в частности, теория сигналов и теория изоморфизма. Как известно, основные исследования автора теории сигналов М. Спенса были посвящены проблеме преодоления неопределенности при принятии инвестиционных решений, в терминологии Спенса - проблеме интерпретации сигналов. Автор теории сигналов утверждал, что теория сигналов наилучшим образом подходит для описания процессов, происходящих на рынках с высокой концентрацией участников. К этому определению, безусловно, можно отнести и процесс коммуникации эмитентов и пользователей отчетности. Схема эмитент-пользователь корпоративной отчетности, по нашему мнению, является абсолютно аналогичной схеме работодатель-кандидат, описанной в исследовании М. Спенса. Если материалами для исследования автора теории сигналов стала информация о образовании, опыте работы, расе, поле и других личностных характеристиках, то в случае с информацией о деятельности компании, речь может идти, например, о таких характеристиках как учетная (в том первичная) информация, нефинансовые показатели и т.д.. Спенс разделял передаваемую в процессе сигнализации, информацию на две категории - первая это «индексы» - это постоянные данные такие как пол, раса и т.д. и, вторая - «сигналы», меняющаяся во времени информация (образование, опыт, возраст и т.п.). В нашем случае, под «индексами» подразумевается стандартная отчетность (минимального обязательного содержания), а «индексы» - это не что иное как дополнительное раскрытие данных о компании, учитывающее актуальные на данный момент времени информационные потребности пользователей. «Сигнальными издержками» М. Спенс называет расходы сигнализатора на изменение качественных характеристик сигнала (например, инвестирование в образование). В случае с корпоративной отчетностью, это могут быть затраты компании на программное обеспечение, лицензирование, создание определенной внутрифирменной инфраструктуры и т.п. Основным отличием сигнальных издержек от прочих расходов является то, что они имеют направленный характер, то есть отвечают конкретным запросам реципиента (требованиям работодателя или пользователя). Предполагается, что все эмитенты пытаются максимизировать разницу между расходами на сигнализацию и предлагаемой выгодой, в данном случае речь может идти о объемах инвестирования, доле рынка, конкурентном преимуществе и т.д.. М. Спенс также писал о цикличности данного процесса, то есть работодатель или, в данном случае, пользователь отчетности с каждым разом повышая требования к кандидатам (эмитентам) тем самым стимулирует производство новых сигнальных издержек (см. рис. 2.). Таким образом, каждый цикл порождает следующий.

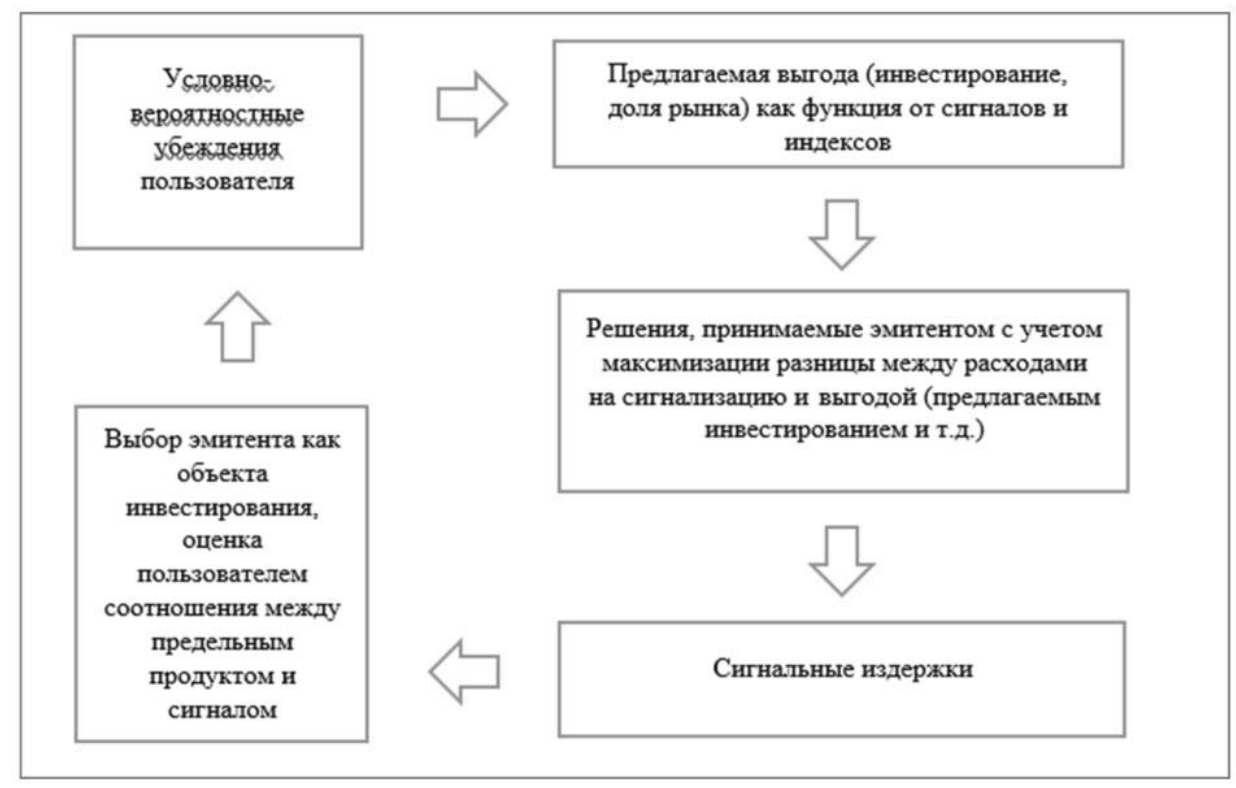

Рис. 2. Схема цикла производства сигнальных издержек Источник: авторская разработка 
В контексте корпоративной отчетности представляется возможным построить модель равновесия интересов пользователей и эмитентов.

Здесь стоит обратить внимание на некоторые особенности данного типа равновесия. Один из них заключается в том, что чем ниже стоимость внедрения, тем большую выгоду получают компании его осуществляющие. Очевидно, что при удешевлении технологий , например, в два раза, как это дано в условии, компаниям явно выгоднее переходить к внедрению новых учетных систем, чем оставаться в категории низкокачественных.

Равновесие в данной модели определяется в контексте «петли обратной связи», то есть ожидания пользователя приводят к определенному уровню доверия в соответствии с «удовлетворяемостью» информационных потребностей пользователя, что, в свою очередь, приводит к инвестициям в развитие учетных систем. После того как компания получила инвестирование, новые информационные потребности пользователей приводят к пересмотру их убеждений относительно содержания отчетности. Здесь цикл начинается снова. Равновесие лучше всего рассматривать как набор убеждений, которые ограничены или, по крайней мере, не противоречит новым данным в конце только что описанного цикла.

Такие убеждения будут иметь тенденцию сохраняться во времени, пока не появятся новые участники рынка готовые удовлетворить новые информационные потребности пользователей и предложить большее раскрытие учетных данных.

При описании данной модели, важно упомянуть об одном существенном допущении - пренебрежении влияния агентской проблемы. В этой связи, важным замечанием о существующем конфликте интересов в процессе сигнализации является высказывание Джозефа Стиглица [39, с. 473], который писал, что участники прочесса делятся на две категории - на тех, кто заинтересован в передаче информачии и на тех, кто заинтересован в том, чтобы информация не передавалась. Однако, при этом, многие авторы исследований в области теории сигналов сходят в общем мнении, что «наличие сигналов безусловно побуждает участников рынка к изменению своего поведения.»

Процесс коммуникации эмитентов отчетности и ее пользователей (наряду с теорией сигналов) может быть также отражен при помощи понятия изоморфизм. Так, А. Хоули описывает изоморфизм как ограничивающий процесс, который вынуждает единицу популяции походить на другие единицы, существующие в условиях той же среды [5, с. 38]. На уровне популяции такой подход предполагает, что организационные характеристики меняются в направлении повышения совместимости с характеристиками внешней среды. В контек- сте развития методов формирования корпоративной отчетности, подтверждением данного тезиса А. Хоули являются результаты, проведенного нами исследования, в частности показавшие существование на практике новых форм персонализированной отчетности, предоставляемой компаниями с целью соответствовать запросам внешней среды, а именно информационным запросам внешних пользователей отчетности.

Очевидно, что компании, работающие в аналогичных условиях будут принимать однородные формы поведения. Такое изоморфное поведение усиливает стабильность и выживание компаний. Следовательно, если наблюдающаяся в последнее время тенденция дезагрегрирования и персонализации отчетных данных и усиление раскрытия информации о компании даст конкурентное преимущество, то это может стать обычной практикой.

\section{Зак^ючение}

В последние годы становится все более заметным факт значимо отличающихся интересов разных групп пользователей отчетности, невозможности игнорирования или ущемления их интересов, а также необходимости гармонизации информационного обеспечения разных групп пользователей учетной информации.

Так, одним из наиболее существенных недостатков, за который подвергаются критике современные модели отчетности является нерепрезентативность, содержащейся в них, информации, возникающая вследствие объединения качественно неоднородных данных, что, в свою очередь, значительно затрудняет получение пользователем актуальной информации о положении дел эмитента отчетности. При этом, следует заметить, что информационное событие ценно лишь тогда, когда оно содержит в себе информацию о новом будущем факте, а в оценке информационного события важно не то, чем оно является в момент регистрации, а то, чем оно может быть в заранее определяемый момент будущего.

На основе полученных данных в ходе исследования были определены следующие направления совершенствования бухгалтерской (финансовой) отчетности:

Первым шагом на пути повышения качества содержащейся в публичной отчетности, информации может быть внедрение систем формирования отчетности в режиме реального времени. Если коммуникационный процесс, как было описано выше, заключается в создании, шифровании, передаче и расшифровке сообщения, то обеспечение доступа к базам учетных данных в режиме реального времени может принципиально изменить процесс коммуникации компаний и пользователей отчетности. 
Вторым важным направлением совершенствования бухгалтерской (финансовой) отчетности может стать ее персонификация. В настоящее время информация все чаще становится предметом экономической деятельности, целью которой является воздействие на потребителя. Речь, в частности, идет о создании технологий обработки «больших данных». В этих условиях персонализированная отчетность может стать важной частью конкурентного преимущества любой компании. Кроме того, персонализированная отчетность в настоящее время является актуальной потребностью пользователей и получает все большее распространение на практике, а объединение баз учетных данных может значительно сократить асимметрию информации на рынке и как следствие снизить риски предприятий реального сектора экономики.

Третьим шагом на пути сокращения информационного разрыва между компаниями и заинтересованными сторонами может стать интерактивный информационный обмен. Как показало исследование, в настоящее время многие компании, чтобы выяснить какая учетная информация интересна пользователям, самостоятельно инициируют диалог с пользователями отчетности, размещая на интернет-сайтах компании соответствующие опросные листы. Однако, следует отметить, что возможно более полезной для пользователей была бы ситуация, когда пользователи сами могли бы инициировать диалог, чтобы задать конкретный вопрос и получить интересующую их информацию. В таком случае пользователи удовлетворяли бы свои информационные потребности, а компании получали сигналы о том, какая именно информация интересует пользователей и соответственно влияет на оценку компании.

Кроме того, необходимым условием эффективного информационного обмена в режиме реального времени является создание соответствующей нормативно-правовой базы для регулирования и легального применения внедряемых учетных систем, а также для удостоверения подлинности предоставляемой учетной информации. Возможно, посредником в таком случае может стать государство.

В ходе исследования, на примере проанализированных запросов различных групп пользователей отчетности было продемонстрировано, что применение современных интегрированных коммуникаций в учете может значительно повысить качество информации, предоставляемой заинтересованным лицам.

Таким образом, существующее положение вещей фактически иллюстрирует сложившееся в настоящее время противоречие между теорией бухгалтерского учета и его нормативным регулированием с одной стороны, и фактической практикой новой корпоративной отчетности с другой. Этот разрыв актуализируется стремительным развитием технологий, принципиально меняющих основание доверия к информации.

При этом следует заметить, что скорость перехода к открытым (частично или полностью) базам данных сегодня зависит большей частью не от возможностей информационных и коммуникационных технологий, а от готовности владельцев баз учетных данных, будь то государство или ТНК, раскрыть информацию и, тем самым, отказаться от прав собственности на нее. Для коммерческих организаций, к тому же, добавляется проблема выбора между конкурентным преимуществом и сохранением коммерческой тайны.

Благодаря тому, что современные интернет-технологии способны обеспечить непрерывное представление учетных данных их пользователям, временной лаг между возникновением событий (ФХЖ) и принятием решений, на их основе, резко сокращается. Это изменение представляется особенно важным так как целью публичной корпоративной отчетности по-прежнему остается обеспечение заинтересованных лиц доступом к информации, необходимой им для принятия обоснованных экономических решений.

Большинство современных пользователей отчетности признают тот факт, что объективность данных существующих форм отчетности является, по большей части, «мифом». Безусловно, речь не идет о преднамеренной фальсификации, а лишь о том, что процесс отбора данных, методы их оценки, по определению, не могут быть абсолютно объективными. Возможно, именно данный факт является причиной результатов исследования, которое показало востребованность персонализированной корпоративной отчетности у различных современных пользователей, а также выявило необходимость внедрения альтернативной вышеописанным формам детализированной отчетности, настраиваемой под каждого отдельно взятого пользователя.

Современные технологии позволяют переключить внимание специалистов с методов регистрации данных на методы их исчисления. Как писал Соколов Я.В. «Принятая методология в большинстве случаев не только не позволяет вскрыть причины хозяйственных процессов и полученных результатов, но и приводит к отождествлению в общих числах (показателях) результатов, полученных за счет внутренних и внешних конъюнктурных факторов». Так, осознание этих недостатков служит основным источником совершенствования учета.

В завершение справедливо будет заметить, что по мере распространения персонализированной отчетности, несомненно, будет расти и список вопросов, связанных с её регулированием. 


\section{ЛИТЕРАТУРА}

1. Блатов Н.А. Балансоведение. Л., Экономическое образование. 1928 / 292 с.

2. Генералова Н.В., Соколова Н.А. Вариативность интерпретации бухгалтерской информации // Вестник СПБГУ. Сер. 5: Статистика и учет. 2012. № 5. С. 175-181.

3. Гребенников В.Ф. Государственная статистика и потребности бизнеса // ЭК0. 2012. № 3. С. 50-69.

4. Дамодаран А. Инвестиционная оценка. Инструменты и методы оценки любых активов. М., Альпина, 2008 / 1950 c.

5. Димаджио П.Дж. Пауэлл У.В. Новый взгляд на «железную клетку»: институциональный изоморфизм и коллективная рациональность в организационных полях // Экономическая социология. 2010. № 1. С. 35-55.

6. Елисеева И.И., Дмитриев А.Л. In memoriam: Ярослав Вячеславович Соколов (1938-2010) - 2-е изд, испр, и доп - СПБ., Нестор-Истрия. 2010 / 508 с.

7. Кащина Ж.Е. Персонализированная корпоративная отчетность: принципы и перспективы развития. Международный бухгалтерский учет. - 2019. - Т. 22. № 2 (452). - С. 147-157.

8. Ковалев В.В. Бухгалтерский учет в России: Смена приоритетов // Вестник СПБГУ. 2010. Вып. 3. С. 98-112.

9. Ковалев В.В., Ковалев Вит. В. Корпоративные финансы и учет: учебное пособие. 3-е изд. М., Проспект. 2015 / 955 с.

10. Львова Д.А. Теория бухгалтерского учета и экономическая теория: в поисках взаимодействия // Финансы и бизнес. 2015. №2. С. 124-142.

11. Мэтьюс М.Р., Перера М.Х.Б. Теория бухгалтерского учета. М., Аудит, ЮНИТИ, 1999 / 663 с.

12. Палий В.Ф. Теория бухгалтерского учета: современные проблемы. М., Бухгалтерский учет, 2007.

13. Плотников В.С., Плотникова 0.В. Философия бухгалтерского учета как экономической науки // Учет. Анализ. Аудит. 2017. №2. С.7-17.

14. Пятов М.Л., Соколова Н.А. Анализ финансовой отчетности. М., Бухгалтерский учет. 2011 / 352 с.

15. Пятов М.Л., Соловей Т.Н. Отчетность компаний в условиях новой информационной реальности // Сборник докладов международного научного форума «0бразование и предпринимательство в Сибири: направления взаимодействия и развитие регионов»: в 4 томах / Новосибирский государственный университет экономики и управления. Н., Новосибирский государственный университет экономики и управления "НИНХ". 2018. С. 208-212.

16. Пятов М.Л. Существует ли наука о бухгалтерском учете? [Эл. ресурс]. URL: https://buh.ru/articles/documents/108216/ (дата 0бращения 15.12.2020).

17. Пятов М.Л., Глинский В.В. Концепция гуманизации данных статистики в работах Я.В. Соколова // Вопросы статистики. 2018. 25(3). С. 79-88.

18. Соколов Я.В. Бухгалтерский учет: от истоков до наших дней: учеб. пособие для вузов. М., Юнити. 1996 / 638 с.

19. Ханин Г.И. Цифры продолжают лукавить (Интервью с Г.И. Ханиным) // ЭК0. 2012. №3. С. 4-13.

20. Хасс Дж. Классика теории организации // Вестник СПБГУ. 2007. Сер 8 Вып 3. С. 112-125.

21. Хендриксен Э.С., Ван Бреда М.Ф. Теория бухгалтерского учета: Пер. с англ./Под ред. проф. Я.В. Соколова. М., Финансы и статистика. $2000 . / 576$ с.

22. Экклз Р. Революция в корпоративной отчетности. М., Олимп-Бизнес. 2002 / 400 с.

23. Aldrich H. (1979) Organizations and Environments. Englewood Cliffs, NJ: Prentice-Hall. 265 p.

24. Ball A., Owen D.L, Gray R. (2000) External transparency or internal capture? The role of third-party statements in adding value to corporate environmental reports. Business Strategy and the Environment. 9(1). Pp. 1-23.

25. Bhimani A., Willcocks L. Digitisation, (2014) "Bigdata” and the transformation of accounting information. Accounting Business Research. 44 (4). Pp. $469-490$.

26. Bystro "m H. (2016) Blockchains, real-time accounting and the future of credit risk modeling. Working paper. Lund, Department of Economics, School of Economics and Management Lund University. $12 \mathrm{p}$.

27. Chambers R.J. (1955) Blueprint for a theory of accounting. Accounting Research. January. Pp. 17-25.

28. Green S., McKinney J.E., Heppard K., Garcia L. (2018) Big Data, digital demand and decision-making. International Journal of Accounting \& Information Management. Vol. 26 Issue: 4. Pp.541-555.

29. Jensen M.C., Meckling W.H. (1976) Theory of the firm: managerial behavior, agency costs and ownership structure. Journal of Financial Economics. 13 (4). Pp. 305-360.

30. KPMG (2010) The Journey to Better Business Reporting. Australia. 25 p.

31. MacNeal K. (1970) Truth in Accounting. Lawrence, Kansas: Scholars Book Co.

32. Mattesich R. (2014) Reality and accounting. London and NY: Routledge. $296 \mathrm{p}$.

33. Sorter George H. (1969) An 'Events' Approach to Basic Accounting Theory. Accounting Review. 44:1. P.13

34. Spence M. (2002) Signaling in retrospect and the informational structure of markets. American Economic Review. Volume 92, Issue 3. Pp. 434-459.

35. Spence A.M. (1973) Time and communication in economic and social interaction. Quarterly Journal of Economics. 87 (4). Pp. 651-660.

36. Spence (1974) M. Competitive and optimal responses to signals: An analysis of efficiency and distribution. Journal of Economic Theory. 7 (3). Pp. $296-332$.

37. Spence M. (1977) Consumer misperceptions, product failure and producer liability. Review of Economic Studies. 44 (3). Pp. 561-571.

38. Stamp E., Mumford M.J., Pesnell K.V. (1993) Philosophical perspectives on accounting. Routledge. London. 324 p.

39. Stiglitz J.E. (2002) Information and the change in the paradigm in economics. American Economic Review. 92. Pp. 460-501.

40. Stuart Cooper, David Crowther and Chris Carter (2001) Challenging the predictiveability of accounting techniques in modelling organizational futures. Management Decision. Vol. 39 № 2, c. 137-146.

41. Sunder S. (2016) Rethinking financial reporting: Standards, norms and institutions. Foundations and Trends in Accounting. Volume 11, Issue 1-2. Pp. 1-118.

42. Wheeler D., Elkington J. (2001) The end of the corporate environmental report? Or The Advent of Cybernetic Sustainability Reporting and Communication. Business Strategy and the Environment. 10(1). Pp. 1-14.

(c) Кащина Жанна Евгеньевна (z.kaschina@lsg.spb.ru). 